\title{
Long-life fluorescent imaging of cells in real time
}

John Haycock, Stanley Botchway, Julia Weinstein, and Gareth Williams

A new family of chemical labels with much longer lifetimes than traditional fluorophores are key to a new biological imaging method: time-resolved emission imaging microscopy.

A fundamental understanding of the mechanisms and processes that control biological systems relies on technological achievements in real-time imaging. A very common imaging modality for studying biological systems is fluorescence microscopy, which benefits from a high spatial and temporal resolution in conjunction with acute sensitivity. ${ }^{1,2}$ A major advantage of fluorescence microscopy is an ability to span magnitudes of length-scale, enabling scientists to visualize whole organisms at one end of the scale right down to single-molecule interactions at the other.

Confocal (and two-photon) microscopy is becoming an increasingly commonplace technique for a multitude of applications, especially for studying cells and tissues in detail. Whatever the application, a fundamental requirement is that of targeting biological entities using stable fluorophores. This is usually achieved by conjugating (joining) the fluorophore directly to an antibody, known as immunofluorescent labeling. Specific biological features can then be identified by the antibody and visualized by exploiting the emissive properties of the fluorophore. ${ }^{3}$

When imaging complex samples such as tissues, nonlabeled structures, including matrix proteins, frequently create unwanted background interference - known as autofluorescencethat 'swamps' the primary signal. This can prevent the acquisition of interpretable images. One way around this would be to make use of differences in the timescale of the emission from the probe and the background. But commercially available fluorophores have inherently very short fluorescence lifetimes (3.6ns, for example, in the case of fluorescein), similar to that of the autofluorescence.

Recently we developed a novel class of luminescent platinum (Pt) (II) labels for biological imaging. ${ }^{4}$ The labels are constructed from a terdentate molecule containing a centrally located

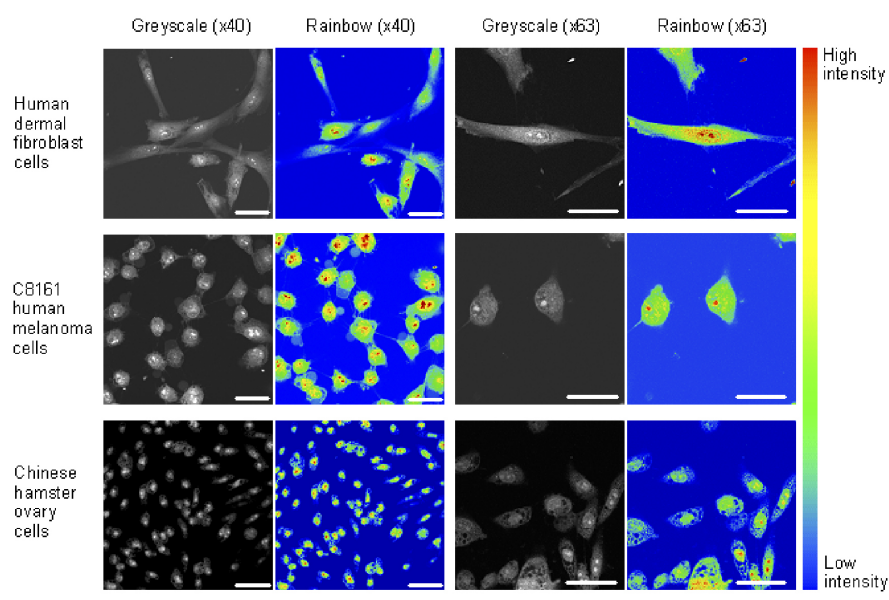

Figure 1. Confocal images of live human fibroblasts, human melanoma cells, and Chinese hamster ovary cells with regions of Pt(II) label accumulation identified. Cells were incubated for 5min, washed with phosphate-buffered saline, and imaged using an excitation wavelength of $488 \mathrm{~nm}$. Scale bar $=10 \mu \mathrm{m}$. (Reprinted with permission. ${ }^{4}$ )

platinum ion flanked by two pyridine rings and a phenyl group. Importantly, the luminescent lifetimes of these molecules are measured in microseconds rather than nanoseconds. The structure of the molecule is important for enabling extended lifetimes. In particular, a carbon-platinum bond at the center of the molecule is key. This very strong but short bond directly contributes to the rigidity of the overall structure and in turn the photostability of the label. Thus, one advantage of these molecules over conventional organic labels is a long emission lifetime due to a triplet excited state rather than a singlet state (as the formally spin-forbidden $S_{0} \leftarrow T_{1}$ transition is promoted by the spin-orbit coupling associated with the platinum atom). In addition, these labels have synthetic versatility (they are both easy to make in the lab and easy to alter) as well as chemical and photochemical robustness. 


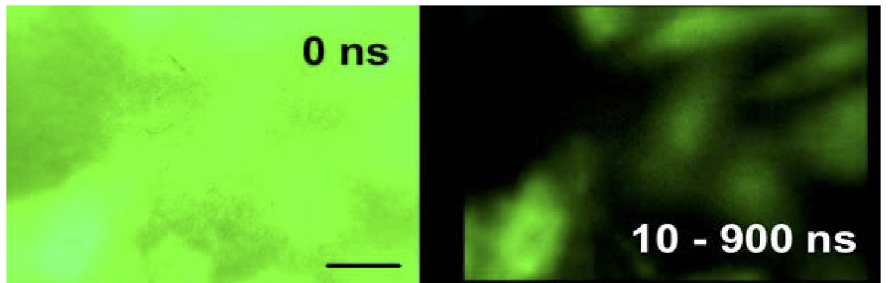

Figure 2. Time-gated cellular imaging. Live CHO cells were labeled with Pt(II) and imaged in the presence of a solution of fluorescein. Images were taken at 0ns (left) and 10ns (right) after a 355nm laserexcitation pulse (pulse length 0.6ns). (Reprinted with permission. ${ }^{4}$ )

Development of $\mathrm{Pt}(\mathrm{II})$ complexes has enabled the realization of a fluorescence-based imaging technique called time-resolved emission imaging microscopy (TREM). TREM is similar to fluorescence lifetime imaging microscopy but uses much longer timescales of hundreds of nanoseconds to microseconds. These timescales offer improved discrimination through the much larger changes in lifetime and allow time-gated experiments to distinguish the labels from short-lived autofluorescence. ${ }^{5}$ TREM does not require fast excitation or detection methods. Indeed, it can be performed with nanosecond lasers and slower gated detectors. However, its development has long been hampered by the lack of cell-permeable, nontoxic luminescent probes with lifetimes in excess of 100ns under ambient conditions.

We have therefore investigated the suitability of $\mathrm{Pt}(\mathrm{II})$ complexes for live cell imaging. 4 We demonstrated that direct labeling of human fibroblasts, human melanoma cells, and Chinese hamster ovary $(\mathrm{CHO})$ cells can be achieved by incubating cells with micromolar concentrations of the labels (see Figure 1). Intracellular accumulation was observed within $5 \mathrm{~min}$ under diffusion control, and the compound was not toxic over a $24 \mathrm{~h}$ period. One- and two-photon excitation revealed specific labeling of the nuclei of all cells as well as a preferential affinity for DNA and RNA structures. Thus, these remarkably stable complexes satisfy the fundamental requirements for TREM, which include a high emission quantum yield (60-70\%), a long emission lifetime, low toxicity, and high cell permeability.

Combining the properties of $\mathrm{Pt}(\mathrm{II})$ labels with TREM makes several exciting applications possible. For example, one can readily discriminate between positively labeled biological structures and background autofluorescence by distinguishing between long- and short-lived species. Figure 2 shows a time-gated cell-imaging experiment in which $\mathrm{CHO}$ cells were labeled with $\mathrm{Pt}(\mathrm{II})$ and imaged in the presence of a solution of fluorescein. Immediately after a laser excitation pulse, an image was taken at 0ns. Little information can be resolved initially from the micrograph because the fluorescein emission has swamped the image. However, the cells can be observed 10ns after the laser excitation pulse due to the $\mathrm{Pt}$ (II) label, which continues to emit light long after the emissive decay of the fluorescein.

In summary, this work shows the power of combining long-lived luminescent probes with TREM for biological imaging and the suitability of this technique for lifetime studies of complex biological structures. We are now generating $\mathrm{Pt}(\mathrm{II})$ conjugated secondary antibodies that will be used in conjunction with monoclonal antibodies for the specific study of biological structures in tissues prone to autofluorescence, in particular skin and nerve.

We thank the UK Science and Technology Facilities Council, Engineering and Physical Sciences Research Council, and Biotechnology and Biological Sciences Research Council for partial support of this work and equipment used.

\section{Author Information}

\section{John Haycock}

Department of Engineering Materials

Kroto Research Institute

University of Sheffield

Sheffield, United Kingdom

http://www.shef.ac.uk/materials/staff/jwhaycock01.html

John Haycock is a reader in bioengineering at the University of Sheffield. He received his PhD in biochemistry from the University of Newcastle (UK), followed by a fellowship at Albany Medical College, NY. His current research is on tissue engineering of nerve and skin, with a particular interest in developing methods for imaging $3 \mathrm{D}$ reconstructed tissues.

\section{Stanley Botchway}

Central Laser Facility

Rutherford Appleton Laboratory

Science and Technology Facilities Council

Oxford, United Kingdom

http:/ / www.clf.rl.ac.uk/Facilities/LSF/LML/contact.htm

Stanley Botchway is a senior scientist and manager of the Laser Microscope Laboratory at the Rutherford Appleton Laboratory. He received his $\mathrm{PhD}$ from Leicester University (UK) followed 
by a fellowship at Harvard Medical School, Boston, MA. His current research is on the use of single- and two-photon excitation for biological imaging.

\section{Julia Weinstein}

Department of Chemistry

University of Sheffield

Sheffield, United Kingdom

http://www.sheffield.ac.uk/chemistry/staff/profiles/

weinstein.html

Julia Weinstein is a lecturer and Engineering and Physical Sciences Research Council Advanced Fellow. She received her PhD from Moscow Lomonosov State University, Russia. Her current research is on chemical approaches to solar energy conversion and the development of time-resolved spectroscopic methods.

\section{Gareth Williams}

Department of Chemistry

University of Durham

Durham, UK

http://www.dur.ac.uk/j.a.g.williams/

Gareth Williams is a senior lecturer at the University of Durham, where he received his $\mathrm{PhD}$. His current research is on the synthesis and properties of light-emitting molecules, both for display screen technology and as luminescent probes for biological imaging.

\section{References}

1. N. Johnsson and K. Johnsson, Chemical tools for biomolecular imaging, ACS Chem. Biol. 2, pp. 31-38, 2007.

2. L. Bachmann, D. M. Zezell, A. D. Ribeiro, L. Gomes, and S. A. Ito, Fluorescence spectroscopy of biological tissues—a review, Appl. Spec. Rev. 41, pp. 575-590, 2006.

3. K. A. Teare, R. G. Pearson, K. M. Shakesheff, and J. W. Haycock, Alpha-MSH inhibits inflammatory signalling in Schwann cells, Neuroreport 15, pp. 493-498, 2004.

4. S. W. Botchway, M. Charnley, J. W. Haycock, A.W. Parker, D. L. Rochester, J. A.Weinstein, and J. A. G. Williams, Time-resolved and two-photon emission imaging microscopy of live cells with inert platinum complexes, Proc. Natl. Acad. Sci. USA 105, pp. 16071-16076, 2008.

5. R. R. de Haas, R. P. M. van Gijlswijk, E. B. van der Tol, J. Veuskens, H. E. van Gijssel, R. B. Tijdens, J. Bonnet, N. P. Verwoerd, and H. J. Tanke, Phosphorescent platinum/palladium coproporphyrins for time-resolved luminescence microscopy, J. Histochem. Cytochem. 47, pp. 183-196, 1999. 